\title{
Diffusionsverhalten von IPBC in wasserbasierten Beschichtungssystemen auf Holzfassaden
}

\author{
Thomas Volkmer • Francis W. M. R. Schwarze
}

Published online: 22 April 2008

(C) Springer-Verlag 2008

\begin{abstract}
Zusammenfassung Wasserbasierte Beschichtungssysteme auf Holzbauteilen sind einem verstärkten Befall durch Schimmel- und Bläuepilze ausgesetzt. Durch einen gezielten Einsatz von Bioziden ist es möglich, einen Befall auf und unter der Beschichtung zu kontrollieren. JodpropinylN-butylcarbamat (IPBC) ist eines der wichtigsten Biozide für den Filmschutz von wasserbasierten Systemen in Europa. In der vorliegenden Arbeit wurde das Diffusionsverhalten von IPBC untersucht. Dabei wurde festgestellt, dass eine Diffusion des IPBC aus der Grundierung in die Deckschicht erfolgt. Die Verteilung des Wirkstoffes in der Deckschicht ist relativ gleichmässig. Die Eindringung in das Holzsubstrat (Fichte) ist mit $250 \mu \mathrm{m}$ sehr gering und die nachgewiesene Wirkstoffmenge in diesem Bereich variiert deutlich. Das Diffusionsverhalten von IPBC ist an einen Wasserdampfstrom und ein notwendiges Konzentrationsgefälle in der Beschichtung gekoppelt. Durch Bewitterungsversuche wurde nachgewiesen, dass bereits nach dreimonatiger Bewitterung nur noch 30\% IPBC in der Deckschicht nachgewiesen werden konnten.
\end{abstract}

\section{Diffusion behavior of IPBC in water based coatings on wooden facades}

Abstract Water based coating systems for wood are more prone to infection and colonization by mould and blue stain

T. Volkmer $(\bullet)$

Berner Fachhochschule,

Solothurnstrasse 102,

2504 Biel, Schweiz

e-mail: Thomas.Volkmer@bfh.ch

F. W.M. R. Schwarze

EMPA,

St. Gallen, Schweiz fungi than systems with organic solvents. The addition of fungicides in coatings inhibits fungal growth on the surface and in the wood. In Europe, IPBC (3-iodo-2-propynyl butylcarbamate) is currently one of the most important fungicides used for film protection. Diffusion analysis of IPBC with HPLC showed that the primer diffused evenly into the topcoat leading to a quite homogeneous distribution. However, it only slightly penetrated $(250 \mu \mathrm{m})$ into the wood and showed a more inhomogeneous distribution in this area. Natural weathering experiments after three months indicate that the amount of IPBC decreased to only $30 \%$ of the original content.

\section{Einleitung}

Wasserbasierte Beschichtungssysteme für Holzbauteile gehören in der Lackindustrie und im Lack verarbeitenden Gewerbe zum heutigen Stand der Technik, obgleich hinsichtlich der Herstellung und der Anwendung noch weiterer Forschungsbedarf besteht (Meijer 2001, Ekstedt 2004), um die bekannten Nachteile gegenüber lösemittelhaltigen Systemen weiter auszugleichen. Bei der Anwendung im Holzbereich für Außenbauteile hat sich die Anfälligkeit gegenüber Mikroorganismen durch den Einsatz wässriger Systeme erhöht, was u.a. auf die notwendigen Additive (Dispergiermittel, Netzmittel, Verdickungsmittel u.a.) für die Herstellung und Stabilisierung der Formulierungen zurückgeführt werden kann. Zudem sind in wässrigen Systemen nur noch spezielle Biozide sinnvoll nutzbar, weil in vielen Fällen die herkömmlichen Wirkstoffe nicht mehr eingesetzt werden können oder nur in deutlich höheren Konzentrationen (z.B. Carbendazim) wirksam sind.

Für wässrige Systeme ist IPBC das weltweit am meisten genutzte Funigzid zum Filmschutz; in Europa wer- 
den jährlich ca. 100 Tonnen umgesetzt (Cook 2002). Es gehört zur Gruppe der Carbamate, welche in erster Linie im Bereich des Pflanzenschutzes Verwendung finden (Lindner 2004). In Kombination mit anderen Wirkstoffen wird IPBC auch im chemischen Holzschutz eingesetzt. Aufgrund seiner geringen Toxizität (LD50 $=1600 \mathrm{mg} / \mathrm{kg}$ bei Ratten), und allergischen Wirkung sowie seines breiten Wirkspektrums gegenüber Pilzen hat es Pentachlorphenol (PCP) auf diesem Gebiet des Biozidmarktes ersetzt (Cook 2002). Trotz seines geringen Gefahrenpotentials als festes Material ist es in Bezug auf das Leben im Wasser toxisch. Die LD50 Dosis für Regenbogenforellen liegt bei $120 \mu \mathrm{g} / \mathrm{L}$. In Bezug auf den Menschen sind keine cancerogenen oder mutagenen Auswirkungen bekannt.

IPBC zeichnet sich bei moderaten Herstellungskosten durch eine hohe Aktivität gegenüber einem breiten Spektrum an Bläue- und Schimmelpilzen aus (einschliesslich $A l$ ternaria spp.). Die geringe UV-Beständigkeit im Film ist in Bezug auf die Persistenz in der Umwelt als vorteilhaft einzuschätzen. Darüber hinaus zeigen neuere Untersuchungen, dass IPBC auch biologisch abbaubar ist, was als Voroder Nachteil gewertet werden kann (Cook et al. 2002). Die teilweise schnelle Auswaschbarkeit von IPBC aus der Beschichtung ist mit Blick auf die toxische Wirkung im Wasser als kritisch zu bewerten.

In der vorgestellten Untersuchung wurden das Diffusionsverhalten und die Beständigkeit von IPBC in wasserbasierten Beschichtungen bzw. im Holz analysiert. In Abhängigkeit verschiedener Alterungsverfahren wurde die Stabilität und die Auslaugbeständigkeit des Wirkstoffes bewertet. Bei den verschiedenen Beschichtungsvarianten wurde der Wirkstoff ausschließlich in der Grundierung eingesetzt und der Nachweis erfolgte entweder in der Deckschicht oder in den oberflächennahen Schichten des Holzes. Somit wurden die beiden möglichen Diffusionswege analysiert.

\section{Material und Methoden}

\subsection{Material}

Die Probebeschichtungen des Holzes setzten sich aus den drei Schichten, Grundierung, Deckschicht 1 und Deckschicht 2, zusammen, wobei Deckschicht 1 und 2 aus demselben Material bestehen. Die einzelnen Schichten wurden manuell auf das geschliffene Substrat appliziert. Für die Grundierung wurden einmal $120 \mathrm{~g} / \mathrm{m}^{2}$ und für die Deckschicht wurde zweimal $100 \mathrm{~g} / \mathrm{m}^{2}$ aufgetragen. Die Zusammensetzung der einzelnen Beschichtungssysteme ist Tabelle $1 \mathrm{zu}$ entnehmen.

Es wurde sowohl in der Grundierung als auch in der Deckschicht nur mit wasserbasierten Systemen gearbeitet. Für die Grundierung wurde ein Hydrosol eingesetzt, basierend auf einer feinstdispergierten Acrylatdispersion mit niedrigem Feststoffgehalt. Für die Deckschicht wurde ein pigmentiertes System auf der Basis des selbstvernetzenden Acrylats XK98 verwendet, welches ein gängiges Bindemittel bei Holzbauteilen im Aussenbereich darstellt. Die Deckbeschichtung war mit herkömmlichen Titandioxidpigmenten ausgerüstet, bei der Variante G kam zusätzlich photokatalytisch aktives Titandioxid zum Einsatz. Das Fungizid IPBC wurde ausschliesslich in die Grundierung eingebracht, dabei wurde mit drei unterschiedlichen Biozidkonzentrationen gearbeitet. IPBC lag für die Einbringung in die Beschichtungsgrundierung in Form verschiedener Biozidprodukte vor. In diesen Biozidprodukten war IPBC entweder zu $10 \%$ in Leinöl oder zu $50 \%$ in Wasser emulgiert (Tabelle 1). Diese Biozidemulsionen wurden durch Rühren in die wasserbasierten Grundierungen eingebracht.

Als Substrat wurde Fichte verwendet. Der Verlauf der Jahrringe im Querschnitt betrug ca. $45^{\circ}$. Die Abmessung der

Tabelle 1 Beschichtungsstoffe - Materialspezifikationen

Table 1 Coating material - Specification

\begin{tabular}{|c|c|c|c|c|c|c|c|}
\hline Variante & $\begin{array}{c}\text { Beschichtungs- } \\
\text { bestandteil }\end{array}$ & $\begin{array}{l}\text { Binde- } \\
\text { mittel }\end{array}$ & $\begin{array}{c}\text { Bindemit- } \\
\text { telanteil }\end{array}$ & $\begin{array}{l}\text { Pigmen- } \\
\text { tierung }\end{array}$ & $\begin{array}{l}\text { Pigment- } \\
\text { anteil }\end{array}$ & $\begin{array}{l}\text { IPBC- } \\
\text { Gehalt }\end{array}$ & $\begin{array}{c}\text { Basisformulieung } \\
\text { des IPBC }\end{array}$ \\
\hline \multirow[t]{2}{*}{$\bar{A}$} & Grundierung & Acrylat & $18 \%$ & - & - & 0,7 & in Leinöl emulgiert \\
\hline & Deckschicht & Acrylat XK 98 & $61 \%$ & Titandioxid & $20 \%$ & & \\
\hline \multirow[t]{2}{*}{ B } & Grundierung & Acrylat & $18 \%$ & - & - & 0,7 & in Wasser emulgiert \\
\hline & Deckschicht & Acrylat XK 98 & $61 \%$ & Titandioxid & $20 \%$ & & \\
\hline \multirow[t]{2}{*}{$\mathrm{C}$} & Grundierung & Acrylat & $18 \%$ & - & - & 0,4 & in Leinöl emulgiert \\
\hline & Deckschicht & Acrylat XK 98 & $61 \%$ & Titandioxid & $20 \%$ & & \\
\hline \multirow[t]{2}{*}{ D } & Grundierung & Acrylat & $18 \%$ & - & - & 0,4 & in Wasser emulgiert \\
\hline & Deckschicht & Acrylat XK 98 & $61 \%$ & Titandioxid & $20 \%$ & & \\
\hline \multirow[t]{2}{*}{$\mathrm{E}$} & Grundierung & Acrylat & $18 \%$ & - & - & 0,2 & in Wasser emulgiert \\
\hline & Deckschicht & Acrylat XK 98 & $61 \%$ & Titandioxid & $20 \%$ & & \\
\hline \multirow[t]{2}{*}{$\mathrm{F}$} & Grundierung & Acrylat & $18 \%$ & - & - & 0,2 & in Leinöl emulgiert \\
\hline & Deckschicht & Acrylat XK 98 & $61 \%$ & Titandioxid & $20 \%$ & & \\
\hline \multirow[t]{2}{*}{ G } & Grundierung & Acrylat & $18 \%$ & - & - & 0,4 & in Leinöl emulgiert \\
\hline & Deckschicht & Acrylat XK 98 & $61 \%$ & $\begin{array}{c}\text { Titandioxid } \\
\text { photokatalytisch } \\
\text { aktives Titandioxid }\end{array}$ & $\begin{array}{l}16 \% \\
4 \%\end{array}$ & & \\
\hline
\end{tabular}


Proben war $375 \times 100 \times 20 \mathrm{~mm}^{3}$. Es wurden pro Variante drei Proben beschichtet. Um die Diffusion des Wirkstoffes besser beurteilen zu können, erfolgte bei ausgewählten Varianten die Applikation zusätzlich auf Fichtefurnier mit einer Dicke von 1, $5 \mathrm{~mm}$.

\subsection{Bewitterung}

Die beschichteten Vollholzproben wurden verschiedenen Bewitterungsverfahren ausgesetzt. Es erfolgte eine Freilandbewitterung über sechs Monate nach EN 927/3 in der Vegetationsperiode von Mai bis Oktober. Dabei wurde im Ausgangszustand sowie nach drei und sechs Monaten eine Analyse des IPBC in der Deckschicht und im Substrat durchgeführt.

Bei den beschichteten Furnierblättern wurde ein definierter Wasserdampfstrom durch die Probe realisiert (ca. $10 \mathrm{ml} / \mathrm{m}^{2} \mathrm{~h}$ ). Der Wasserdampf bewegte sich durch das Furnier über die Grundierung in die Deckschicht (siehe Abb. 1). Der Nachweis der Anreicherung des IPBC erfolgte in der Deckschicht der Beschichtung. Durch diese Versuchsanordnung sollte nachgewiesen werden, dass das Biozid in der Grundierung nicht fest verankert ist und durch den Einfluss von dampfförmigem Wasser im Holz und der Beschichtung transportiert werden kann. Darüber hinaus kann davon ausgegangen werden, dass bei einer Bewitterung der Oberfläche von der Beschichtungsseite sich das Wasser hinter der Beschichtung im Holz ansammelt und

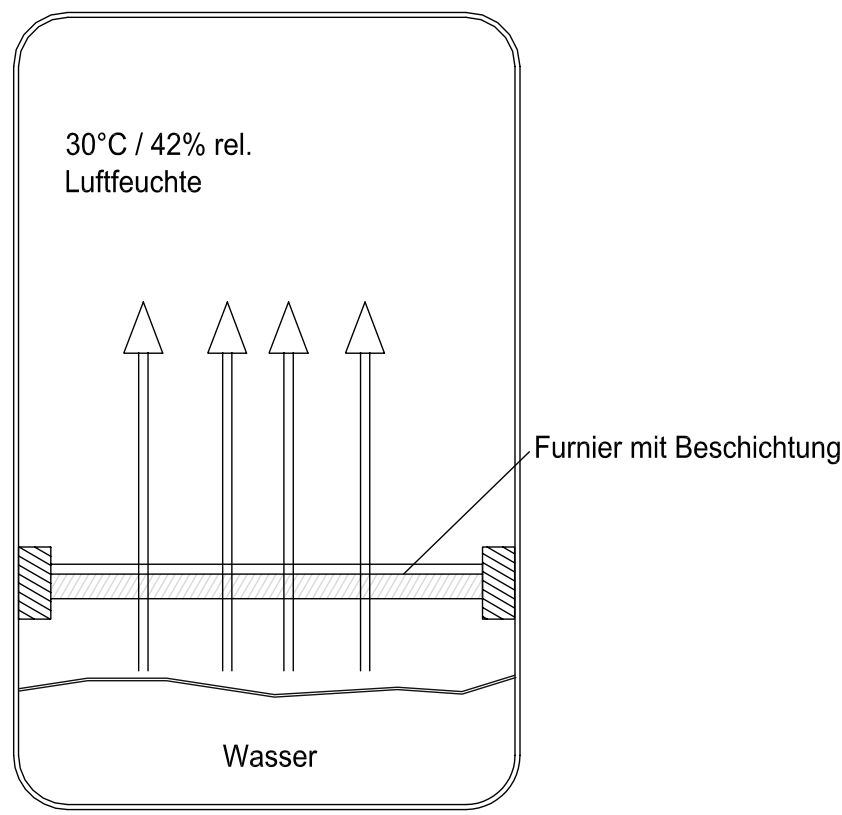

Abb. 1 Prinzipskizze für die Durchführung des Diffusionsversuches durch das beschichtete Furnier

Fig. 1 Sketch of the diffusion experiment through the coated veneer bei einer folgenden Trockenperiode dann wiederum durch die Beschichtung in die Umwelt entweicht. Generell sollte mit dem verwendeten Versuchsdesign der Transport des IPBC an sich analysiert werden. Aufgrund der Vielzahl an Fassadenaufbauten und Konstruktionen kann durch den gewählten Versuchsaufbau nur eingeschränkt auf das Verhalten des IPBC bei einer konkreten Fassadenanwendung geschlossen werden.

\subsection{Analyse}

Die Analyse des IPBC erfolgte mit Hilfe der HPLC im ,,reversed mode“ im Gradientenverfahren (siehe Tabelle 2). Es wurde mit einer C18-Säule (Nucleosil hochdicht gepackt) gearbeitet. Als Eluentenflüssigkeit kam ein Gemisch aus Acetonitril und Wasser zum Einsatz. Es wurde ein Probenvolumen von $10 \mu \mathrm{l}$ eingespritzt und mit einem Fluss von $0,9 \mathrm{ml} / \mathrm{min}$ gearbeitet. Die Retensionszeit für IPBC betrug unter diesen Bedingungen 8,1 min. Die Detektierung erfolgte mit einem UV-DA (Diodenarray)-Detektor bei $195 \mathrm{~nm}$ und einer Bandbreite von $8 \mathrm{~nm}$. Die Kalibrierung des Systems erfolgte aufgrund der zu erwartenden Messwerte mit zwei Kalibrierreihen mit je drei Wiederholungen:

Reihe $1: 26,36,52,64,72 \mathrm{mg} / \mathrm{l}$ und

Reihe $2: 1,2,3,4,5 \mathrm{mg} / 1$.

Die Bestimmungsgrenze wurde entsprechend dem kleinsten Kalibrierwert auf $1 \mathrm{mg} / \mathrm{l}$ festgelegt, was je nach Einwaagemenge durchschnittlich 0, 004 Masse-\% bezogen auf das Lackvolumen entspricht.

Die Wiederfindungsrate von IPBC wurde unter Verwendung des Deckschichtmaterials durchgeführt. Dazu wurde eine definierte Menge an Biozid in das flüssige Deckschichtmaterial eingebracht und anschliessend auf Glas appliziert. Nach erfolgter Trocknung wurde das Beschichtungsmaterial abgetragen und analysiert. Die Wiederfindungsrate lag zwischen 87-92 Masse-\% bezogen auf das Lackvolumen.

Für die Analyse der Deckschicht wurde das Beschichtungsmaterial mechanisch mittels einer Ziehklinge von der

Tabelle 2 Gradient des Eluentengemischs während der Flüssigchromatographie

Table 2 Gradient of the eluente mixture during the fluid chromatography

\begin{tabular}{lcc}
\hline Zeit in min & Acetonitril in $\%$ & Wasser in $\%$ \\
\hline 0 & 55 & 45 \\
1 & 55 & 45 \\
7,5 & 60 & 40 \\
10 & 88 & 12 \\
15 & 88 & 12 \\
21 & 55 & 45 \\
25 & 55 & 45 \\
\hline
\end{tabular}


Oberfläche entfernt und in einem Gemisch aus Acetonitril und Wasser (Mischungsverhältnis von 60/40 Volumen-\%) extrahiert. Die Proben wurde bei $20^{\circ} \mathrm{C} 20 \mathrm{~min}$ lang gelagert und danach 30 Sekunden lang auf einem Vorteks der Firma Merk geschüttelt. Danach wurde die Probe bei $6000 \mathrm{Umdr} / \mathrm{min}$ für eine Dauer von 5 min abfiltriert. Aufgrund der manuellen Probengewinnung variierte die Probenmenge der abgetragenen Lackschicht und lag für die verschiedenen Testreihen im Bereich von 80-150 mg. Die Probeneinwaage wurde mit $4 \mathrm{ml}$ Lösemittelgemisch versetzt. Aufgrund des präparativen Aufwandes wurde nur für die Variante F eine Mehrfachbestimmung des IPBC Gehaltes vor der Bewitterung an fünf verschiedenen Stellen der beschichteten Probe durchgeführt, um eine Tendenz für die Verteilung des Wirkstoffes in der Deckschicht abzuleiten.

Die Bestimmung des IPBC im Beschichtungsmaterial erfolgte an zwei Stellen immer in der Deckschicht, einerseits direkt an der Oberfläche der Beschichtung und andererseits im Bereich, der das Substrat berührt (siehe Abb. 2).

Für die Bestimmung der Eindringung und des Abbaus von IPBC im Substrat wurde das Holz schichtenweise $(50 \mu \mathrm{m})$ mit einem Mikrotom abgetragen und in Gruppen zusammengefasst $(0-250 \mu \mathrm{m}, 250-1000 \mu \mathrm{m}, 1000-2000 \mu \mathrm{m}$, 2000-3000 $\mu \mathrm{m})$. Für die Analyse ergaben sich daraus Einwaagemengen von 100-200 mg, welche mit $8 \mathrm{ml}$ Lösungsmittel versetzt wurden. Die Extraktion erfolgte dann analog dem Deckschichtmaterial. Da anzunehmen war, dass der Wirkstoff, welcher im Biozidprodukt in Wasser emulgiert war, tiefer in das Holz eindringt, erfolgte für diese Varianten die Analyse bis in eine Tiefe von $3 \mathrm{~mm}$. Bei den Varianten, bei denen der Wirkstoff im Biozidprodukt in Leinöl emulgiert war, wurde die Analyse der Eindringtiefe bis in einen Bereich von $1 \mathrm{~mm}$ durchgeführt. Nur für Variante B wurde an 12 verschiedenen Stellen der Probe vor der Bewitterung

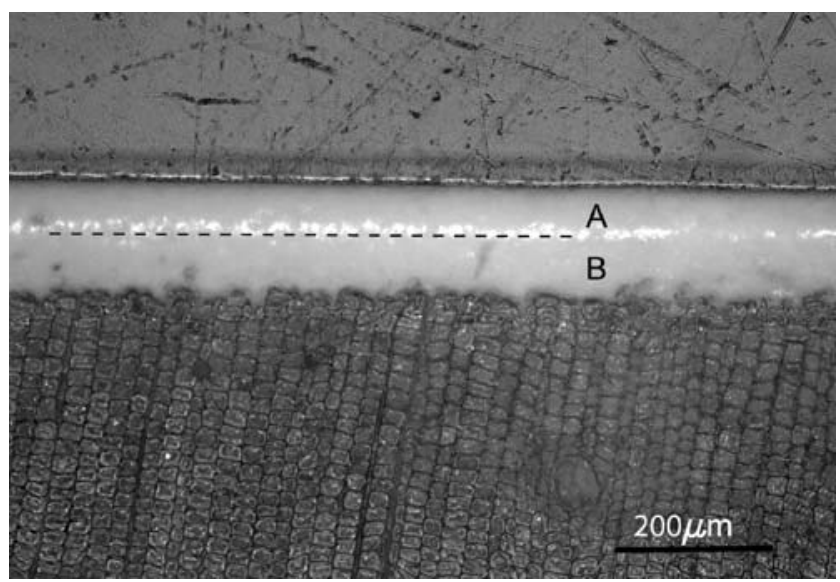

Abb. 2 Analysebereiche in der Deckschicht: A Analyse an der Oberfläche, B Analyse im Deckschichtbereich in Substratnähe

Fig. 2 Analytic areas in the topcoat: A analysis near the surface of the topcoat, B analysis of the topcoat close to the substrate in einem Tiefenbereich von 0-250 $\mu \mathrm{m}$ eine Bestimmung des IPBC-Gehaltes durchgeführt, um eine Aussage über die Verteilung des Wirkstoffes in diesem Tiefenbereich machen zu können.

\section{Ergebnisse}

Der Diffusionsversuch mittels eines definierten Dampfstroms durch die Beschichtung zeigte, dass in Abhängigkeit des Biozidgehaltes in der Grundierung der Wasserdampfstrom das IPBC aus der Grundierung herauslöst und in die Deckschicht transportiert (Abb. 3). Je mehr IPBC in die Grundierung eingebracht wird, umso mehr kann in die Deckschicht diffundieren. Da IPBC nur einen Dampfdruck von $2,4 \times 10^{-4} \mathrm{~Pa}$ aufweist (siehe DGfH-Merkblatt 2004), muss die Bewegung des Biozids auf die Wasserdampfflüchtigkeit zurückgeführt werden. Wenn an der Oberfläche keine äußeren Einflussfaktoren, wie z.B. UVLicht, fliessendes Wasser, Wasserdampf oder Bakterien, wirksam sind, reichert sich das IPBC entsprechend dem durchgeführten Diffusionsversuch an und wird nicht abgebaut oder abtransportiert. Unabhängig vom Wasserdampfstrom bewegt sich im Ausgangszustand bereits eine gewisse Menge des IPBC in die Deckschicht (Abb. 3 und 6 (Referenzproben)). Es ist zu vermuten, dass einerseits das Konzentrationsgefälle zwischen Grundierung und Deckschicht und andererseits das Herauslösen des Biozids aus der Grundierung während der Applikation der Deckschicht zu diesem Stofftransport führt.

Die Bewitterungszeit hatte einen deutlichen Einfluss auf den Abbau oder den Abtransport des IPBC aus der Beschichtung (Abb. 6). Bereits nach dreimonatiger Bewitte-

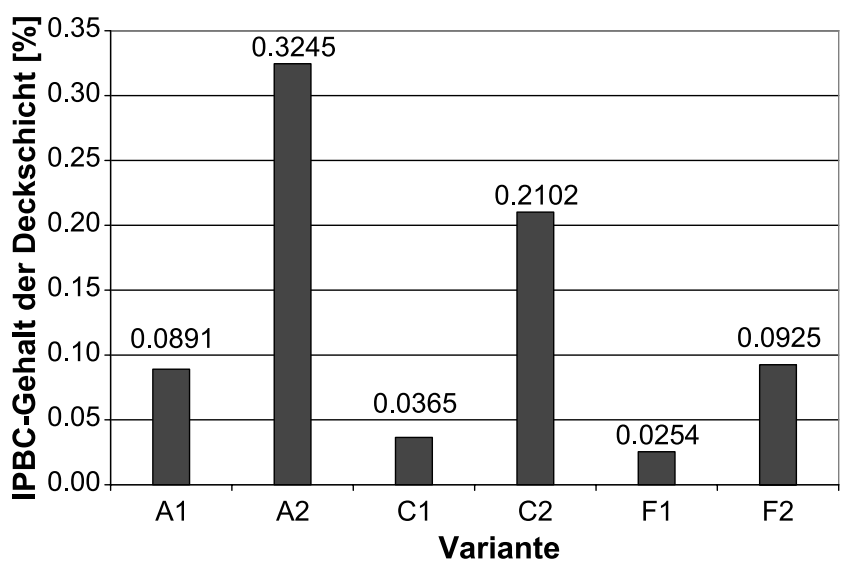

Abb. 3 IPBC-Gehalt (Masse-\%) in der Deckschicht (A1, B1, F1 vor Dampfstrom, A2, B2, F2 nach Dampfstrom, Biozidgehalte der Grundierung: A1, A2 0,7\%, B1, B2 0,4\% F1, F2 0,2\% IPBC)

Fig. 3 IPBC-content (weight-\%) in the topcoat (A1, B1, F1 before vapor flow, A2, B2, F2 after vapor flow, biocide content of the primer: $\mathrm{A} 1, \mathrm{~A} 20.7 \%, \mathrm{~B} 1, \mathrm{~B} 20.4 \%$ F1, F2 0.2\% IPBC) 


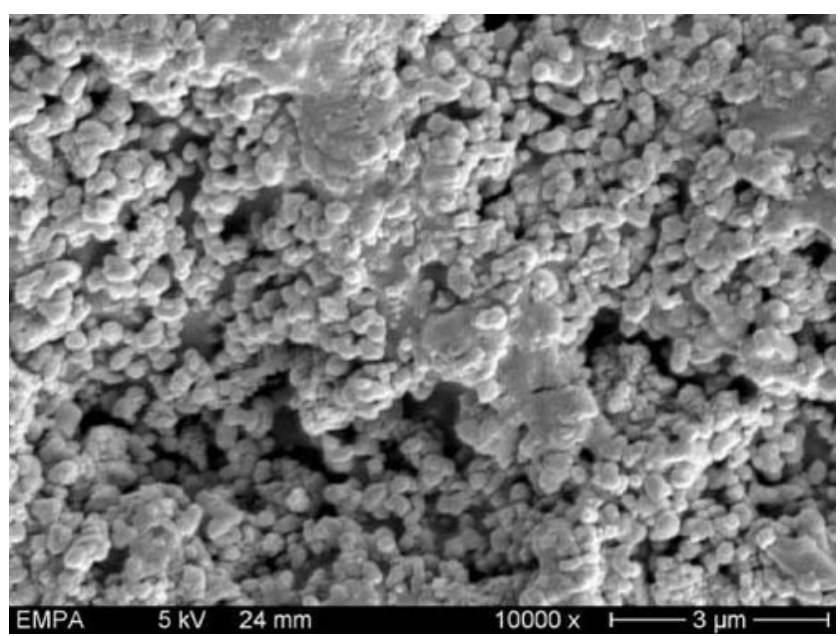

Abb. 4 Deckschicht Variante $G$ mit photokatalytisch aktivem Titandioxid nach 6 Monaten Freilandbewitterung, starker Abbau des Bindemittels und freiliegende Pigmente

Fig. 4 Topcoat of variant $G$ with photo-catalytic active Titan-dioxide after 6 months exposure to natural weathering. Note: significant degradation of the binder and free pigments

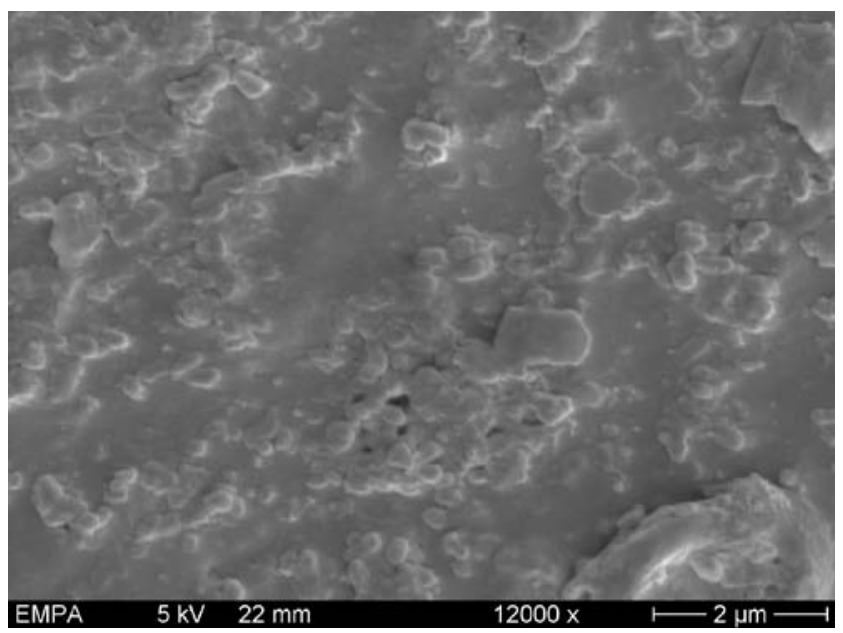

Abb. 5 Deckschicht Variante A selbstvernetzendes Acrylat XK98 nach 6 Monaten Freilandbewitterung, geringer Abbau des Bindemittels

Fig. 5 Topcoat of variant A (self-cross linking acrylate XK 98) after 6 months exposure to natural weathering. Note: only slightly degradation of the binder

rung (Mai-Juli 2006) zeigte sich ein deutlicher Rückgang des Biozidgehaltes an der Oberfläche. Bei allen Varianten ohne photokatalytisch aktives Titandioxid (Varianten A-F) konnte nach dieser Zeit nur noch ein Restgehalt von weniger als 50\% des Wirkstoffes nachgewiesen werden. Dabei kann davon ausgegangen werden, dass mehr als die analysierten Mengen an Wirkstoff abtransportiert oder abgebaut wurden, da aufgrund der Bewegung des Wirkstoffes praktisch immer IPBC aus der Grundierung an die Oberfläche gewandert ist. Bei Variante $\mathrm{G}$, bei der mit $4 \%$ photokataly-

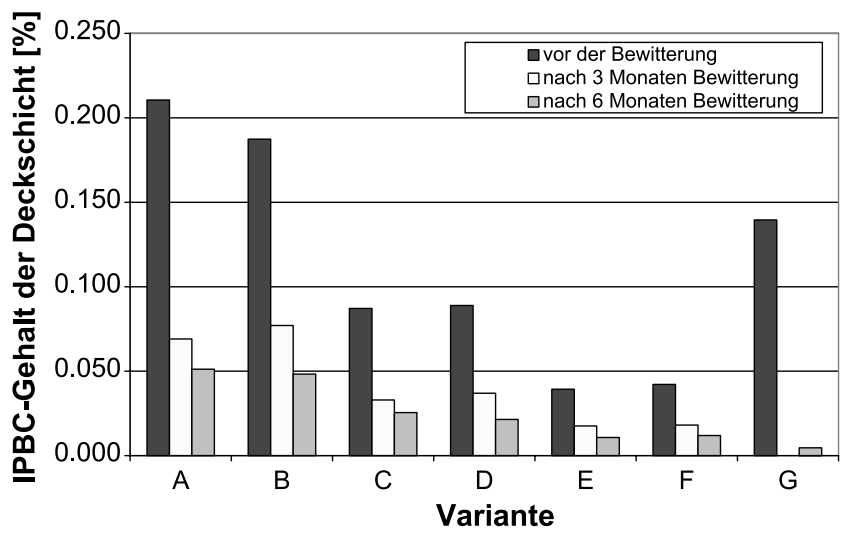

Abb. 6 IPBC-Gehalt (Masse-\%) in der Deckschicht in Abhängigkeit der Bewitterungsdauer

Fig. 6 IPBC-content (weight-\%) in the topcoat in relation to the weathering time

tischem Titandioxid gearbeitet wurde und welche ansonsten die gleiche Formulierung wie Variante $\mathrm{C}$ aufwies, war nach drei Monaten kein IPBC entsprechend der Bestimmungsgrenze von 0,004 Masse-\% mehr nachzuweisen. Es ist anzunehmen, dass die photochemischen Prozesse sowie der Einfluss von Feuchtigkeit an der Deckschichtoberfläche zu diesem Abbau und/oder Abtransportes des Biozids geführt haben. Dies wurde durch die Auswertung der rasterelektronischen Aufnahmen bestätigt. Im Verlauf der Bewitterung ist ein deutlicher Abbau des Bindemittels erkennbar, was sich offensichtlich auch auf die Biozidkonzentration ausgewirkt hat (vgl. Abb. 4 und 5).

Nach sechsmonatiger Bewitterung zeigt sich, dass sich der IPBC-Gehalt in der Deckschicht stabilisiert. Der Konzentrationsrückgang war geringer als in den ersten drei Bewitterungsmonaten. Das macht deutlich, dass relativ hohe Biozidanteile (Varianten A und B) sensibler auf die äußeren Umweltfaktoren (UV-Strahlung und Feuchtigkeit) reagieren und dass der Biozidabbau generell mit zunehmender Zeit und abnehmender Konzentration langsamer abläuft. In Bezug auf Variante $\mathrm{G}$ ist bemerkenswert, dass nach sechsmonatiger Bewitterung wieder eine geringe Menge an IPBC nachgewiesen werden konnte, was die oben gemachten Ausführungen bestätigt, wonach es im Verlauf der Bewitterung immer wieder zu einem Stoffstrom aus der Grundierung in die Oberfläche kommt. Je nach Witterungseinflüssen erfolgt dann der Abbau oder Abtransport des IPBC oder es kommt zeitlich begrenzt zu einer Anreicherung an der Oberfläche.

Die Diffusion und die niedrigen gemessenen Restgehalte des IPBC an der Oberfläche sind in Zusammenhang mit der Verteilung des Wirkstoffes in der Deckschicht zu sehen. In Tabelle 3 sind die Ergebnisse des Biozidnachweises für Variante $F$ an fünf verschiedenen Probenstellen in der Deckschicht im Ausgangszustand zu sehen. Daraus ist zu erkennen, dass die mengenmäßige Variation des Biozides 
Tabelle 3 IPBC-Verteilung in der Deckschicht vor der Bewitterung

Table 3 IPBC-distribution in the topcoat prior to weathering

in der Deckschicht mit einem Variationskoeffizienten von $21,43 \%$ relativ hoch ist. Es ist davon auszugehen, dass die Beschichtung im Ausgangszustand als homogene Schicht betrachtet werden kann und die Variation des Biozidgehaltes auf die Verteilung des Wirkstoffes im Holz zurückzuführen ist. Es ist anzunehmen, dass die strukturellen Merkmale bei Fichte, wie Jahrringaufbau (Früh- und Spätholz) und Tüpfelverschluss, die Eindringung des Biozides stark beeinflussen und somit auch der Biozidgehalt in der Deckschicht entsprechend differiert.

Die IPBC-Analyse in der Grenzschicht zeigte (Abb. 7), dass es in diesem Bereich bis auf Variante $\mathrm{A}$ zu einer Anreicherung des Wirkstoffes gekommen ist. Dabei ist die mögliche Streuung der Wirkstoffverteilung zu berücksichtigen (Tabelle 3). Die Ergebnisse legen nahe, dass die Verteilung des Wirkstoffes im Beschichtungsquerschnitt eher homogen ist, was dem gleichmäßigen Aufbau der Beschichtung entspricht. Trotzdem ist erstaunlich, dass es infolge des einseitigen Biozidstromes nur zu einem geringen Biozidgefälle kommt. Es ist anzunehmen, dass auftretende Konzentrationsunterschiede in der Deckschicht über den gekoppelten Dampf-/Biozidstrom aus der Grundierung permanent ausgeglichen werden.

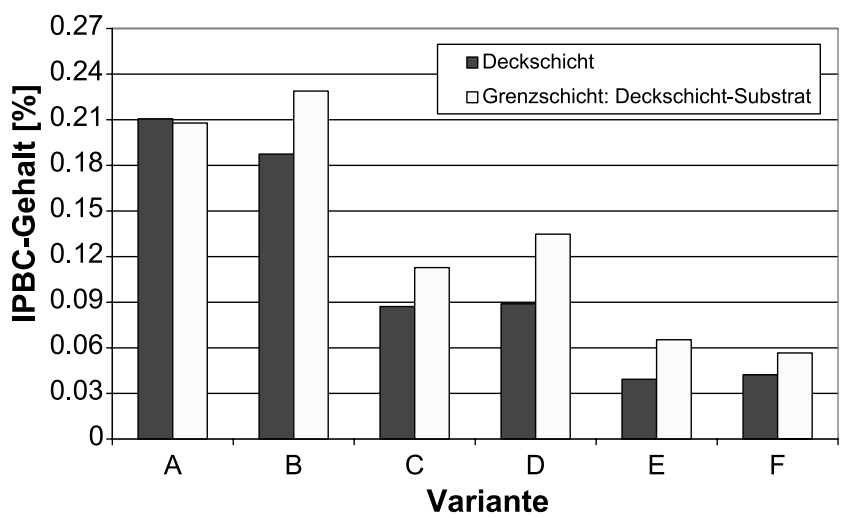

Abb. 7 IPBC-Gehalt (Masse-\%) der Deckschicht im Ausgangszustand an der Beschichtungsoberfläche und im Grenzbereich zwischen Holz und Beschichtung

Fig. 7 IPBC-content (weight-\%) in the upper layer of the topcoat and in the interface between topcoat and wood substrate before exposure to weathering

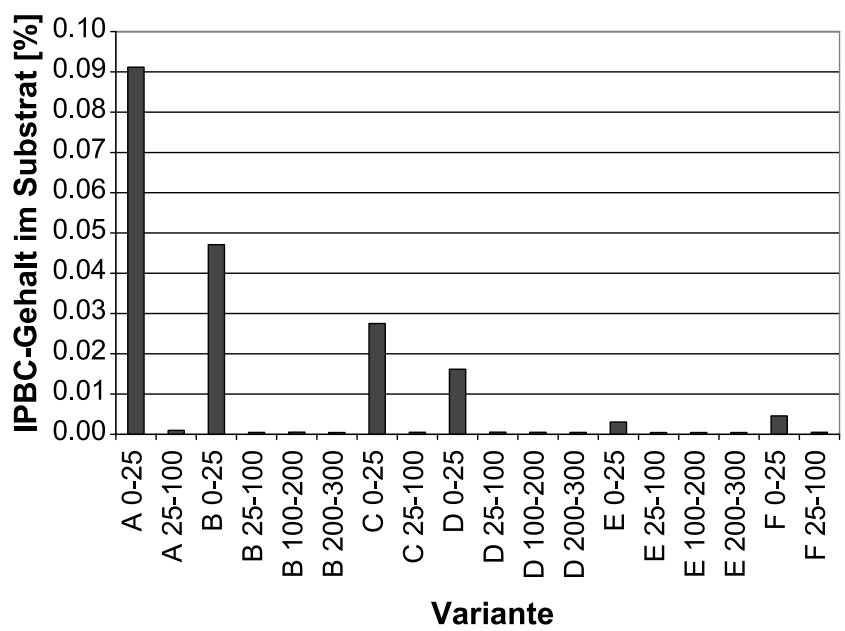

Abb. 8 IPBC-Gehalt (Masse-\%) im Substrat in Abhängigkeit des IPBC-Gehaltes der Grundierung und des Abstandes von der Holzoberfläche vor der Bewitterung (Biozidgehalte der Grundierung: A $0,7 \%$, B $0,7 \%$, C $0,4 \%$, D $0,4 \%$, E $0,2 \%$, F $0,2 \%$ IPBC)

Variantenbezeichnung: A 0-25 Variante A analysierter Tiefenbereich $0-250 \mu \mathrm{m}$

Fig. 8 IPBC-content (weight-\%) in the wood substrate in relation to the IPBC-content of the primer and the distance from the wood surface prior to weathering (biocid content of the primer: A $0.7 \%, \mathrm{~B}$ $0.7 \%$, C $0.4 \%$, D $0.4 \%$, E $0.2 \%$, F $0.2 \%$ IPBC)

Variant designation: A 0-25 variant A section analyzed in a depth of $0-250 \mu \mathrm{m}$

Die Analyse von IPBC im Substrat wurde entsprechend der vorgestellten Methode in verschiedenen Schichten des Holzes beginnend von der Oberfläche durchgeführt. Dabei stellte sich heraus, dass der Wirkstoff vor der Bewitterung praktisch nur sehr wenig in das Holz eindringt. Für alle Varianten konnte die Substanz nur im Bereich von 0-250 $\mu \mathrm{m}$ Tiefe nachgewiesen werden (Abb. 8), was belegt, dass Fichte mit wässrigen Substanzen schlecht tränkbar ist.

Die Eindringtiefe konnte nicht mit der Biozidgrundformulierung in Zusammenhang gebracht werden, d.h. dass kein Einfluss auf die Eindringung nachgewiesen werden konnte, ob der Wirkstoff im Biozidprodukt in Wasser oder in Leinöl emulgiert war. Tendenziell war bei den Varianten mit einem höheren Biozidgehalt in der Grundierung auch mehr Wirkstoff im Holz nachweisbar.
Tabelle 4 IPBC-Verteilung im Substrat im Tiefenbereich von $0-250 \mu \mathrm{m}$ vor der Bewitterung Table 4 IPBC-distribution in the substrate in a depth area from $0-250 \mu \mathrm{m}$ prior to weathering

\begin{tabular}{lccccc}
\hline $\begin{array}{l}\text { Variante/Proben- } \\
\text { entnahmestellen }\end{array}$ & $\begin{array}{c}\text { Mittelwert } \\
{[\%]}\end{array}$ & $\begin{array}{c}\text { Standardabwei- } \\
\text { chung [\%] }\end{array}$ & $\begin{array}{c}\text { Variations- } \\
\text { koeffizient [\%] }\end{array}$ & $\begin{array}{c}\text { Maximum } \\
{[\%]}\end{array}$ & $\begin{array}{c}\text { Minimum } \\
{[\%]}\end{array}$ \\
\hline $\mathrm{B} / 12$ & 0,062 & 0,04 & 64,5 & 0,16 & 0,02 \\
\hline
\end{tabular}


Die Streuung der Wirkstoffverteilung im Holz war deutlich höher als die Verteilung des Biozides in der Beschichtung, was durch den Vergleich von Tabelle 3 mit Tabelle 4 deutlich wird. Die stark schwankende Penetration von wässrigen Substanzen bei Fichte wird dabei durch folgende Parameter beeinflusst: Tüpfelverschluss der Tracheiden im Verlauf der Holztrocknung, Dichteunterschiede im Jahrring und das manuelle Applikationsverfahren.

\section{Diskussion}

Die Mobilität des Biozides IPBC in der Beschichtung wurde anhand verschiedener Analysen nachgewiesen. Es erfolgt eine Diffusion aus der Grundierung in die Deckschicht und in das Substrat. In Abhängigkeit vom IPBC-Gehalt der Grundierung wandert unterschiedlich viel Wirkstoff in die Deckschicht.

Es ist davon auszugehen, dass zwei Mechanismen für die Bewegung der Substanz im Beschichtungssystem verantwortlich sind.

Der Konzentrationsunterschied zwischen den einzelnen Schichten (Grundierung und Deckschicht) forciert eine Bewegung des Biozides, so dass sich ein Konzentrationsausgleich einstellt. Die Stärke des Diffusionsstromes ist dabei von der Grösse des Konzentrationsgefälles abhängig. In dieser Arbeit konnte nicht abschließend geklärt werden, ob dieser Ausgleich auch in Zusammenhang mit der Applikation der Deckschicht erfolgt oder im Verlaufe der Zeit kontinuierlich verläuft. Es wäre möglich, dass die Aufbringung der wasserlöslichen Deckschicht bereits einen Teil des Biozides aus der getrockneten Grundierung wieder herauslöst und somit der Beginn des Konzentrationsausgleiches initiiert wird. Demgegenüber ist zu beachten, dass in der getrockneten Deckschicht selbst keine wesentlichen Konzentrationsunterschiede festgestellt wurden. Die Deckschicht wurde auf die Grundierung zweimal appliziert und bildet danach einen zusammenhängenden Film. Es wäre zu vermuten gewesen, dass im Bereich der ersten Deckschicht (direkt auf dem Substrat) deutlich mehr Biozid nachgewiesen werden kann, was aber nicht der Fall war. Deshalb ist davon auszugehen, dass sich der Prozess des Konzentrationsausgleiches in Abhängigkeit von der Zeit gleichmäßig über die Beschichtung vollzieht und weniger durch den Applikationsprozess der wässrigen Deckschicht beeinflusst wird.

Ein weiterer Faktor für die Biozidbewegung im Beschichtungssystem ist das Vorhandensein von Wasser. Dabei ist zu beachten, dass die Diffusion des Wirkstoffes auf der Wasserdampfflüchtigkeit beruhen muss, da IPBC nicht destillierbar ist und nur in sehr geringem Umfang wasserlöslich ist $(156 \mathrm{mg} / \mathrm{l})$. Die Bewegung des Wassers durch die Beschichtung vollzieht sich dabei in Form einer Diffu- sion im Konzentrationsgefälle nach Fick (Bagda 1986) und nicht als Fluss durch eventuelle Öffnungen in der Beschichtung (Poren, welche durch die gesamte Beschichtungsdicke verlaufen, sind nicht vorhanden).

Dementsprechend ist davon auszugehen, dass die Wassermoleküle infolge eines gerichteten Dampfstromes teilweise Biozidmoleküle „mit sich reißen“ und es so zu der Bewegung des IPBC in der Beschichtung kommt. Der sich einstellende Biozidstrom ist dabei abhängig vom Konzentrationsgefälle und der Intensität des Wasserdampfstromes. Dabei ist es möglich, dass es zu einer Anreicherung des Wirkstoffes an der Oberfläche kommt, wie die Ergebnisse dieser Arbeit gezeigt haben.

Die oben genannten Prinzipien des Biozidtransportes, speziell die Bewegung mit dem Wasser, sind für organische Biozide teilweise bekannt (Callow et al. 1995, Landesumweltamt Nordrhein Westfalen 2005) und stellen die Basis für deren Wirksamkeit einerseits und einen möglichen Abbaumechanismus andererseits dar.

In Bezug auf den IPBC-Abbau wurde weiter festgestellt, dass in Abhängigkeit von der Konzentration der Grundierung bereits nach sechsmonatiger Freilandbewitterung nur noch vergleichsweise geringe Mengen an Wirkstoff nachgewiesen werden konnten. Dieser schnelle Verlust von IPBC stellt für die Anwendung in der Praxis ein Problem dar. Die geringe UV-Stabilität des Wirkstoffes ist seit längerem bekannt (Lindner 2004) und konnte bis jetzt nicht gelöst werden. Lee et al. (1991a,b) weisen nach, dass IPBC durch die energiereiche UV-Strahlung unter Jodabspaltung photolytisch zu Prop-2-inyl-N-butylcarbamat (PBC) umgewandelt wird und dabei weitestgehend seine Wirksamkeit verliert. Nach Cook et al. (2002) beträgt die biozide Wirkung des Transformationsproduktes PBC nur noch 1/1000 im Vergleich zu IPBC.

Zur Stabilisierung des IPBC in der Beschichtung ist zu empfehlen, herkömmliche UV-Schutzmittel (Absorber oder Radikalfänger) direkt in die Biozidbasisformulierung mit einzuarbeiten und nicht in das Bindemittel. Durch die räumliche Nähe zwischen Biozidmolekül und UV-Absorber ist zu vermuten, dass die Absorbtion der UV-Strahlung in Bezug auf IPBC effizienter abläuft und das Biozid weniger schnell abgebaut wird. Das würde der Studie des Landesumweltamtes Nordrhein Westfalen von 2005 entsprechen, wonach die Auswaschbarkeit und der Abbau verschiedener Biozide über die Formulierung der Farbe gesteuert werden kann.

Nach Cook (2002) kann der Abbau von IPBC auch durch Bakterien verursacht werden. Dabei erfolgt ähnlich wie bei der UV-Strahlung eine Abspaltung des Jods und es entsteht das Prop-2-inyl-N-butylcarbamat (PBC) mit deutlich reduzierter Wirksamkeit. Mehrere Arbeiten (O’Neil 1988, Souza und Gaylarde 1996) weisen auf die Präsenz von Bakterien auf Fassadenoberflächen hin, wodurch die Möglichkeit ei- 
nes bakteriellen Abbaus des IPBC in Fassadenbeschichtungen gegeben ist.

Die genannten Mechanismen in Bezug auf die Reduktion des IPBC-Gehaltes (Auswaschung, Photolyse, bakterielle Transformation) erklären die geringen Restgehalte des Wirkstoffes in den Beschichtungen. Es ist davon auszugehen, dass die Mechanismen als Kombination wirken und der Abbau oder Abtransport sich dadurch noch verstärken. Eine Vorhersage des Biozidgehaltes in der Beschichtung ist nach den Ergebnissen dieser Arbeit in begrenztem Umfang möglich, aber die Komplexität des Abbaus infolge der Freibewitterung bleibt ungeklärt.

Die Ergebnisse für den Nachweis von IPBC im Substrat zeigen deutlich, dass wässrige Systeme prinzipiell nur sehr wenig in das Fichtensubstrat eindringen (Rijckaert et al. 2001, Grüll et al. 2002) und demzufolge auch nur wenig Wirkstoff in das Substrat transportiert wird. Vor der Bewitterung erfolgte die Penetration bis in eine Tiefe von ca. $250 \mu \mathrm{m}$. Die Streuung der Einbringmenge ist dabei relativ hoch, was in Zusammenhang mit den anatomischen Merkmalen von Fichte zu sehen ist (Tüpfelverschluss, Dichteunterschiede: Frühholz/Spätholz).

Quellen zur analytischen Bestimmung der Eindringung von IPBC in Fichte standen zum momentanen Zeitpunkt nicht zur Verfügung, weshalb ein quantitativer Vergleich nicht möglich war. Bei einer Parallelstudie an der EMPA St. Gallen (Landmesser und Schwarze 2007) wurde anhand eines biologischen Nachweises die Eindringtiefe vergleichbarer Proben auf Kiefernsplintholz untersucht. Dabei wurden Eindringtiefen von 1-2 mm ermittelt. Grüll et al. (2002) ermitteln über biologische Tests für eine wasserbasierte Grundierung mit 0,8\% IPBC Eindringtiefen in Kiefernsplintholz von bis zu $2 \mathrm{~mm}$ und stellen dabei fest, dass die Erhöhung der Einbringmenge nicht linear mit der Eindringtiefe korreliert.

Die analysierten Einbringmengen und die Werte für die Eindringtiefe aus der Literatur belegen, dass IPBC in einer wässrigen Dispersion nur wenig (Kiefernsplintholz) bis sehr wenig (Fichtenholz) in das Substrat eindringt. Andere organische Wirkstoffe dringen deutlich tiefer ein. Schoknecht und Bergmann (2000) ermitteln analytisch nach einem 24 Stunden dauerenden Tauchprozess für eine 11\%ige Benzalkoniumchloridlösung Eindringtiefen auf Kiefernsplintholz bis $6 \mathrm{~mm}$. Die Konzentration der Lösung war dabei entsprechend der vorgegebenen Einbringmenge eingestellt. Grüll et al. (2002) zeigten, dass Tebuconazol in einem Lösemittel gelöst bis zu $7 \mathrm{~mm}$ in Kiefernsplintholz eindringen kann.

Die genannten Fakten machen deutlich, dass die Eindringung des IPBC nicht ausschließlich durch das Holzsubstrat limitiert ist. Der Wirkstoff selbst sowie dessen Konzentration sind mit entscheidend für die Eindringtiefe.

Der biozide Wirkstoff in der Grundierung hat die Aufgabe das Substrat (und bei entsprechenden Diffusionsei- genschaften auch die Deckschicht) vor einem biologischen Befall zu schützen. Ein Befall durch Mikroorganismen (speziell Basidiomyzeten) ist aber nur ab einer dauerhaften Holzfeuchte von über $20 \%$ zu erwarten (Erler 2002). Da die Eindringung der wässrigen Substanzen bei Fichte nur sehr oberflächig erfolgt, bedeutet dies aber auch, dass das Substrat gegen eine Durchfeuchtung gut geschützt ist. Die geringe Eindringtiefe des IPBC kann von diesem Standpunkt her auch positiv gesehen werden. Eine Erhöhung der Eindringtiefe der Substanz ist zwangsläufig an eine bessere Eindringung von Wasser gekoppelt, was aber eine Durchfeuchtung des Substrates bedeutet und somit die Anfälligkeit gegenüber einem Befall durch Mikroorganismen erhöht.

\section{Schlussfolgerung}

Mittels der HPLC-Methode konnte die Diffusion des Wirkstoffes IPBC aus einer wasserbasierten Grundierung in die Deckschicht und in das Substrat nachgewiesen werden. Dabei kommt es im Beschichtungsmaterial als auch im Holzsubstrat zu einer Anreicherung des Wirkstoffes. Die Eindringung von IPBC in Fichtenholz ist sehr gering und variiert stark. Die Basisformulierung des Biozidproduktes (Wasser- oder Leinölemulsion) hat keinen nachweisbaren Einfluss auf die Diffusionsvorgänge im Beschichtungssystem.

IPBC wird in der Deckschicht im Verlauf der Bewitterung sehr schnell abgebaut oder abtransportiert, was in erster Linie auf die kombinierte Belastung aus UV-Strahlung und anschließende Auswaschung zurückgeführt wird. Nach sechsmonatiger Freilandbewitterung waren bei den untersuchten Proben max. 30\% Restgehalt des Wirkstoffes nachzuweisen. In Bezug auf das Substrat führt die Bewitterung zu einem tieferen Eindringen der Substanz in das Holz.

\section{Literatur}

Callow ME, Heaton PE, Butler GM (1995) Control of Mould Growth by antifungal Paint, Biodeterioration \& Biodegradation 9, ISBN: 085295319 4, pp 567-573

Bagda E (1986) Zur Wasserdampfdiffusion in Beschichtungsstoffen. Farbe Lack 92(4):276-279

Cook SR (2002) The bacterial biotransformation of the organic antisapstain biocide IPBC, Doctoral Thesis, Department of Biology, University of London

Cook SR, Sullivan J, Dickinson DJ (2002) The bacterial Biotransformation of IPBC. Annual Meeting of the international Research Group on Wood Preservation, Stockholm

DGfH Merkblatt (2004) Gaschromatografische Bestimmung des Gehaltes an 3-Jod-2-propinylbutylcarbamat im Bereich von 50$500 \mathrm{mg} / \mathrm{kgHolz}$ nach Extraktion mittels Methanol. http://www. dgfh.de/Frameset-Publikation-Download.html 
Ekstedt J (2004) Current views on the role of water in the behavior of coated wood substrates, Cost E 18 Proceedings EU Seminar 26.-27.4.2004, Paris

EN 927 (2006) Beschichtungsstoffe - Beschichtungsstoffe und Beschichtungssysteme für Holz im Außenbereich - Teil 3: Freibewitterung

Erler K (2002) Holz im Außenbereich. Birkhäuserverlag, Basel Boston Berlin

Grüll G, Janotta O, Melzer H (2002) Protective primeres against brown and rot fungi: Influence of the amount of application on the depth of efficacy, Proceedings Sonderseminar Cost E18 „Biologischer Abbau von beschichtetem und unbeschichtetem Holz Beschichtungen und Trägermaterial“", Lissabon

Landesumweltamt Nordrhein Westfalen (2005) Gewässerbelastung durch den Eintrag von Bioziden aus Dachfarben - eine Risikoabschätzung. Merkblätter Band 51

Landmesser H, Schwarze FWMR (2007) Abschlussbericht KTI Projekt/Teil 1: Entwicklung eines fungizidarmen Beschichtungssystems unter Berücksichtigung des integrierten Holzschutzes. EMPA, St. Gallen

Lee DH, Tsunoda K, Takahashi M (1991a) Photostability of organoiodine wood preservatives. 1. Progressive degradation and loss in fungal inhibition rate through photoirradiation. Mokuzai Gakkaishi 37:76-81

Lee DH, Tsunoda K, Takahashi M (1991b) Photostability of organoiodine wood preservatives. 2 . The photolytic process of preservatives. Mokuzai Gakkaishi 37:261-265

Lindner W (2004) Surface Coatings in: Directory of microbiocides for the Protection of Materials by Paulus W. Chapman \& Hall, London

Meijer de M, Thurich K, Militz H (2001) Quantitative measurements of capillary coating penetration in relation to wood and coating property. Holz Roh- Werkst 59/1-2:35-45

O’Neil TB (1988) Succession and interrelationships of microorganism on painted surfaces. Int Biodeter Biodegr 24:373-379

Rijckaert V, Stevens M, Acker v J (2001) Effect of some formulation parameters on the penetration and adhesion of water borne primers into wood. Holz Roh- Werkst 59(5):344-350

Schoknecht U, Bergmann H (2000) Eindringtiefenbestimmung für Holzschutzmittelwirkstoffe. Holz Roh- Werkst 58:380-386

Souza de, Gaylarde CC (1996) Biodeterioration of Varnished Wood and its Control, 10th International Biodeterioration and Biodegradation Symposium. Dechema Monographs Vol. 133, Frankfurt Weinheim 